\title{
Design of Reliable Architecture for Testing Timed Behavior of OSI Protocols
}

\author{
Kshirasagar Naik \\ Computer Networks Laboratory, School of Computer Science and Eng., University of \\ Aizu, Aizu-Wakamatsu City, Fukushima, 965 JAPAN, k-naik@u-aizu.ac.jp
}

Keyword Codes: C.2.1; D.2.5

Keywords: Network Architecture and Design; Testing

\begin{abstract}
There are two contributions of this paper. First, we introduce the notion of a timeserver protocol in a test architecture to dynamically estimate the round-trip network delay in the service provider. The generalization capability of neural networks is used as the central idea in the dynamic estimation of round-trip delays from actual measurement of delays in the past. Second, we define the notion of architecture reliability and the properties a reliable architecture must satisfy. The notion of a filter protocol is introduced to obtain reliability in a test architecture. Finally, we combine the time-server and filter protocols with a basic architecture to obtain a reliable test architecture.
\end{abstract}

\section{Introduction}

In OSI (Open Systems Interconnection) [ISO 9646] protocol testing, a test architecture defines an interconnection of a Lower Tester (LT), an Upper Tester (UT), and an Implementation Under Test (IUT) using Points of Control and Observations (PCO) and a service provider network. The reactive nature of communication protocols [NASA 92] coupled with real-time behavior makes protocol testing a difficult task. Research on the following two main components of protocol testing can reduce this difficulty: (i) formally designing test cases from protocol specifications, and (ii) reliably executing the test cases using parameters of the run-time environment.

Algorithmic generation of test cases has received much attention [DSU 90]. However, the topic of reliably executing test cases has received very little attention. Executing a test case in a reliable manner means isolating the result of executing a test case from previous execution of test cases. Using a run-time environment for test execution involves selecting an appropriate subset of the entire test suite, parameterizing the selected test cases, obtaining the executable versions of the parameterized test cases, and estimating 
values for timers in test cases.

Timers play an important role in the proper functioning of communication protocols. Therefore, test suites contain several test cases to check timed behavior of protocol implementations. An important activity in testing timed behavior is the estimation of values of timers in test cases. At present, timer durations are statically chosen at the beginning of test execution. However, selection of timer durations cannot be a static activity, because of the fact that message delay is a dynamic activity that depends on the network load of the underlying service networks and the speed of the systems on which the test and implementation entities run. Selecting values for timers while executing test cases is a non-trivial task due to two main obstacles: (i) round trip delay between the lower tester and the IUT, and (ii) the IUT's reaction time upon receiving a stimulus or the expiration of a timer [ALKA 90]. Therefore, there is a need for research to dynamically compute timer durations.

In the external test architectures [ISO 9646], the Lower Tester and the IUT communicate through an underlying service provider which may use a chain of public data networks. In case of a third-party testing and in case of a central laboratory-based testing, the use of public data networks in the conformance testing process will be very common [ALKA 90]. Thus, it is important to study the side-effects produced by the service provider networks on conformance testing.

Because of the unreliable nature of an IUT, all the events sent by the LT may not be acknowledged by the IUT, the IUT may crash during the execution of a test case leaving many events from the LT unacknowledged, and orderly closing of a connection may not be possible. That means, in a data network connecting the LT and the IUT, spurious messages can keep moving from the source (LT or IUT) to the destination (IUT or LT) in both directions of a defunct connection. If care is not taken in test architectures, these events from one connection or test case will affect the result of testing the IUT using a re-run of the same test case or a new test case. This problem will become more serious in two instances: first, in case of automated selection and execution of test cases from a database of hundreds of test cases; second, in case of high-speed protocols, in which hundreds of protocol data units are in transit, abortion of a connection between the LT and IUT will lead to the generation of many spurious events. Though protocols are supposed to handle spurious events, the occurrence of spurious events in the testing process is highly undesirable due to the fact that an IUT may behave incorrectly and spurious events would cause confusion in identifying whether an undesired behavior is actually caused by a faulty IUT.

The contribution of this paper is two-fold. First, we introduce the concept of a time server protocol to dynamically estimate the round-trip delay in the service provider. Dynamic estimation of delay is done using the generalization capability of neural networks [WASS 89] from samples of actual delays measured in the past. Second, in order to design a reliable test architecture we introduce the notion of reliability in test architectures and discuss a filter protocol to achieve it. Finally, we combine the above two concepts to obtain a reliable test architecture suitable for testing time related behavior of communication protocols.

In Section 2, we discuss the role of timers in protocols. In Section 3, we present a brief introduction to neural networks and the detailed design of a time-server protocol to 


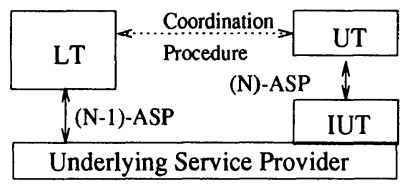

Figure 1: DS Test Architecture

dynamically estimate a timeout interval. In Section 4, we discuss architecture reliability. In Section 5, we put together the ideas in Section 3 and 4 to obtain a reliable architecture suitable for testing timed behavior of OSI protocols.

\section{Test Architectures and Timeout Intervals}

\subsection{Test Architectures}

In the OSI test execution framework [ISO 9646], the four basic architectures are Local Single-layer (LS), Coordinated Single-layer (CS), Distributed Single-layer (DS), and Remote Single-layer (RS). The last three are called external architectures. As an example, the DS architecture is shown in Fig. 1. The importance of external test architectures is due to the fact that conformance testing services can also be provided by international organizations and commercial test laboratories. In addition, the external test architectures have the advantage of closely resembling a more realistic communication environment than the local architecture.

The three conceptual components of a test case are test purpose, dynamic behavior, and test verdict. A test purpose is a high-level description of the protocol function to be tested by a test case. The dynamic behavior is designed to achieve what is stated in the high-level test purpose. At the end of executing a test sequence, a test verdict is assigned to the behavior of the IUT with respect to the test purpose. If the behavior of the IUT is allowed by the protocol specification and the test purpose is satisfied, then the test case assigns a Pass verdict to the IUT. If the behavior of the IUT is not allowed by the protocol, then the test case assigns a Fail verdict. However, if the behavior of the IUT is allowed by the protocol specification, but the test purpose is not satisfied, then the test case assigns an Inconclusive verdict. Therefore, the above three components of a test case are intertwined. The idea of test verdicts will be used to explain the concept of a reliable test architecture in Section 4.

\subsection{Timer and Timeout Interval}

A. Necessity of a timer: In computer networks, a timer is a failure detection mechanism which is used to decide when to retransmit a lost message, or when to abandon a possibly broken connection. Conceptually, a timer is an alarm clock which goes off immediately after a specified timeout period. The timeout period of a timer has two directly conflicting implications on the functioning of a communicating entity that uses the timer: early failure detection and reducing the number of false alarms. Hypothetically, a timer should go off as soon as there is some kind of system failure which a timer is 
supposed to indicate. However, since a timer is initiated to go off after a predetermined timeout period, designing a timer that goes off as soon as a failure occurs is equivalent to predicting a component failure in a system, which is an unsolvable problem. Therefore, in practice a timeout may occur much after a failure has occurred and may occur without the occurrence of any failure. The second occurrence of a timeout is referred to as a false alarm. Thus, while computing the timeout period of a timer, a balance must be struck between the conflicting requirements of early failure detection and the necessity to reduce the number of false alarms.

B. Different Types of Timers: Timers are used to report various kinds of probable failures with each kind of failure depending on different kinds of environmental effects. That is, an objective can be attached with each timer. Examples of some timer objectives are detection of temporary message loss, detection of a permanent channel breakdown, and detection of a remote host dead. While a timer is initiated, estimation of its timeout interval must be based on the objective of the timer.

C. Problems with Estimating a Timeout Interval: Since a timer is used based on the notion that messages have finite propagation delays, estimation of a timeout interval must be done based on the up-to-date information available about the delay characteristic of the network. Thus, estimating a timeout interval involves understanding the running environment of the communication system [ZHAN 86, ALKA 90]. Estimation of a timeout period becomes increasingly difficult when two entities communicate through a variety of media, such as high speed LANs, narrow bandwidth dial-up lines, long delay satellite channels, and reliable long haul networks, under various network loads. Computing a timeout interval in an adaptive manner is not an easy task. There are four factors affecting the round trip time of a message in a heterogeneous service provider: (i) message length, (ii) dynamic network routing, (iii) network load, and (iv) performance criterion.

\section{Dynamic Computation of Timeout Interval}

A timeout interval $T_{o}$ is computed as $T_{o}=2 * T_{p}+T_{c}$ where $T_{p}$ is the expected propagation delay between the source and the destination and $T_{c}$ is the expected computation time for the destination to generate a message expected by the source. Estimation of $T_{c}$ is dependent on factors such as the protocol policy to send a message and the speed of computation at the destination. However, estimation of $T_{p}$ depends on the network load in the service provider connecting the source (LT) and the destination (IUT). In this section, we present the concept of a time server protocol that dynamically estimates the message propagation delay between two communicating entities using the generalization capability of neural networks from a set of measured network delays.

\subsection{Neural Networks}

Three important properties of neural networks are [WASS 89]: (i) they learn from experience, that is, they are trained, rather than programmed, with input data samples, (ii) they generalize their behavior from previous examples to new ones when new data are presented to them, and (iii) they abstract essential characteristics from inputs containing irrelevant data. Shown a set of inputs, perhaps with desired outputs, they self-adjust to 


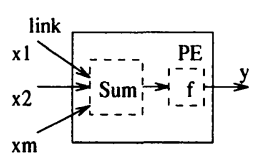

(a)

Legend:

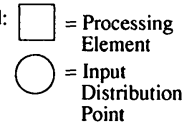

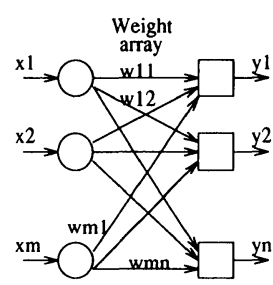

(b)

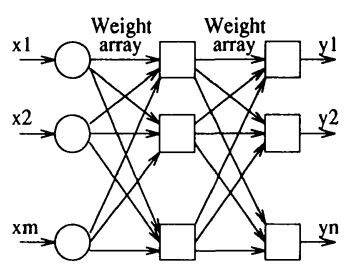

(c)

Figure 2: (a) Single neuron, (b) Single-layer network, (c) Two-layer network

produce consistent responses. In the following, we give brief introductions to organization of neural networks, the kinds of algorithms used to train the networks, and some applications of neural networks.

A. Organization of neural networks: A neural network consists of neurons and links. Neurons can be considered as the processing elements (PEs) in the network and a link is the data path between two neurons. All links are weighted, so that data is multiplied by the link weight when it passes through the link. The basic operation of a neuron can be seen in Fig. 2(a). Each neuron sums all its inputs and passes the result through an activation function $f$. Typical activation functions are hard limiter, threshold logic, and sigmoid function. Although a single neuron can perform certain simple pattern detection functions, the power of neural computation comes from connecting neurons into networks. Examples of single-layer and two-layer networks are shown in Figs. 2(b) and (c), respectively. Those networks are feedforward or nonrecurrent in the sense that there are no feedback paths in the networks. More general networks that contain feedback connections are said to be recurrent. A neural network is used in two phases. In the first phase, the network is trained using a set of example patterns and in the second phase, the network is recalled using an input pattern.

B. Training of neural networks: A network is trained so that application of a set of inputs produces the desired set of outputs. Each such input (or output) is called a vector. Training is accomplished by sequentially applying input vectors, while adjusting network weights in an algorithmic manner. During training, the network weights gradually converge to values such that each input vector produces the desired output vector. Training algorithms are categorized as supervised and unsupervised.

Supervised training requires the pairing of each input vector with a target vector representing the desired output; together these are called a training pair. A network is trained over a number of such training pairs $\left\{\left(X_{i}, Y_{i}\right) \mid 1 \leq i \leq N\right\}$ using the following four steps after initializing the weights $\mathrm{W}$ :

Step1: Execute Step2 and Step3 for all $i$ and go to Step4 to continue or to stop.

Step2: Apply $X_{i}$ to the network and compute the actual output $Y_{a}$ and error $E_{i}=Y_{i}-Y_{a}$. Step3: Feed the error $E_{i}$ back through the network and update the weights W.

Step4: If the sum of mean square errors $E_{i}>\epsilon$, then goto Step 2, else stop. Updation of the weights W in Step 3 are done using various rules [RUHI 86, HECH 88, 


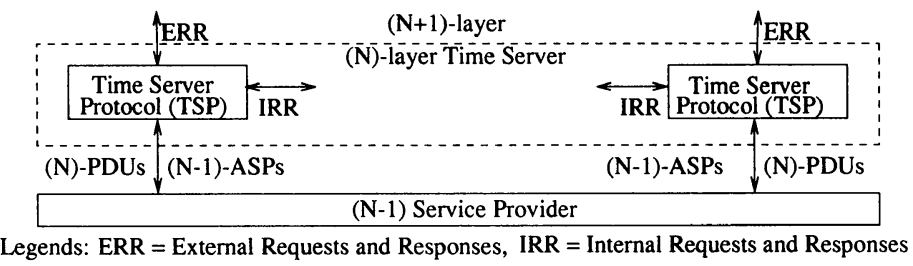

Figure 3: The Concept of a Time Server Protocol

WASS 89] depending on the structure of the network.

Unsupervised training requires no target vector for the outputs. The training set consists solely of input vectors. The training algorithm modifies network weights to produce output vectors that are consistent, that is, both application of one of the training vectors or application of a vector that is sufficiently similar to it will produce the same pattern of outputs. The training process, therefore, extracts the statistical properties of the training set and groups similar vectors into classes. Unsupervised training is largely used in classifying patterns into classes [KOHO 88].

C. Recall of data: After a network is trained using a set of training pairs $\left\{\left(X_{i}, Y_{i}\right) \mid 1 \leq\right.$ $i \leq N\}$ or a set of training patterns $\left\{X_{i} \mid 1 \leq i \leq N\right\}$, depending on whether the training is supervised or unsupervised, data can be recalled from the network at its output by presenting an input $X_{j}$ to the network.

D. Application of neural networks: Two main areas of applications of neural networks are pattern recognition and classification [GROS 88] and learning a mathematical function [HECH 88]. To make a neural network learn a mathematical function, one generates a set of examples $\left(x_{1}, y_{1}\right),\left(x_{2}, y_{2}\right), \ldots$ of the action of the desired function $\phi$, where $y_{i}=\phi\left(x_{i}\right)$ or $y_{i}=\phi\left(x_{i}\right)+n$, where $n$ is a noise process. These examples statistically define the desired input/output relationship. The example set $\left\{\left(x_{i}, y_{i}\right)\right\}$ is used to train a network that learns the function $\phi$. In the recall phase, by presenting a value $x_{j}$ to the network, we obtain the value $y_{j}$ at the network's output, where $y_{j}=\phi\left(x_{j}\right)$.

E. Using neural network in this paper: In this paper, by using a neural network we represent the delay characteristic of a service provider by a mathematical function. We measure samples of actual delay in the service provider and use the pairs $\left\{\left(T_{i}, d_{i}\right)\right\}$, where $d_{i}$ is the measured delay in the service provider at instant $T_{i}$, to train a neural network. Then, we recall the neural network to estimate delays in the future. To obtain the best estimate of the current network delay from the measurement of a samples of delays in the past, we measure the service provider delays and train/recall the neural network in a dynamic manner as explained in Section 3.2.

\subsection{Time Server Protocol}

The conceptual operation of the Time Server Protocol (TSP) is shown in Fig. 3. While testing a $(\mathrm{N})$-layer protocol, we need two (N)-layer TSP entities communicating through the same $(\mathrm{N}-1)$-service provider used in the testing process. This allows the TSP to estimate the propagation delay in the service provider as accurately as possible. 


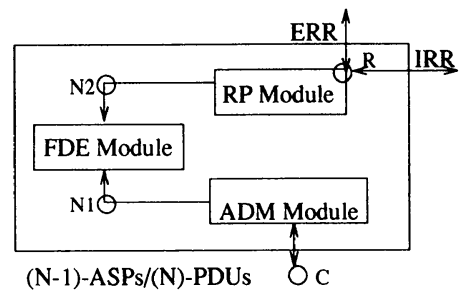

\author{
Legends: \\ $\mathrm{RP}=$ Request Processing \\ $\mathrm{FDE}=$ Future Delay Estimation \\ $\mathrm{ADM}=$ Actual Delay Measurement \\ $\mathrm{O}=$ Interaction Points \\ N1,N2,C,R = Names of Interaction Points
}

Figure 4: Modular Structure of a Time Server Protocol

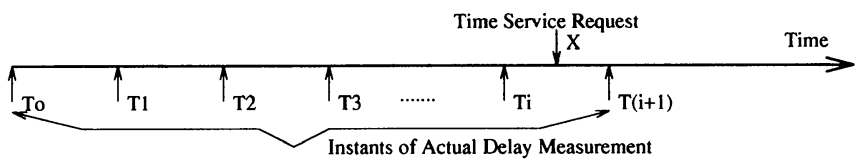

Figure 5: Conceptual Interactions between ADM and FDE Modules

A TSP entity communicates with the service provider using the set of abstract service primitives supported by the service provider and provides service to entities in $(\mathrm{N})$-layer and $(\mathrm{N}+1)$-layer. Since, while testing a $(\mathrm{N})$-layer IUT in the external test architectures, the Lower Tester runs as a $(\mathrm{N})$-entity protocol and the Upper Tester runs as a $(\mathrm{N}+1)$ entity protocol, a (N)-layer TSP provides service to the entities of both $(\mathrm{N})$ - and $(\mathrm{N}+1)$ layer. In Section 5, we show the details of the use of the TSP protocol in the context of our new test architecture.

The TSP entity, shown in Fig. 4, consists of three modules: Actual Delay Measurement (ADM) Module, Future Delay Estimation (FDE) Module, and Request Processing (RP) Module. The interactions among the three modules are explained using Fig. 5. The ADM module communicates with the underlying service provider through an interaction point $C$ and with the FDE module through the interaction point $N 1$. The RP module communicates with the FDE module through the interaction point $N 2$ and with the users through $R$. The ADM module, by communicating with another ADM module at a remote site through the service provider, shown in Fig. 3, measures the actual message propagation delays $\left\{d_{0}, d_{1}, \ldots, d_{i}, d_{i+1}, \ldots\right\}$ in the service provider at time instants $\left\{T_{0}, T_{1}, \ldots, T_{i}, T_{i+1}, \ldots\right\}$, respectively. After computing a pair $\left(i, d_{i}\right)$, the ADM module sends the pair $\left(i, d_{i}\right)$ to the FDE module. In a later part of this section, we will discuss how the ADE module computes a $\left(i, d_{i}\right)$ pair. The FDE module, after receiving a $\left(i, d_{i}\right)$ pair, incrementally updates the estimation of future propagation delay in the service provider using a neural computation technique. The RP module receives requests for the current best estimation of the propagation delay in the underlying service provider from the Upper Tester or the Lower Tester. When the RP module receives such a request at time $X$, the FDE module returns an estimation of the propagation delay computed until the largest $T_{i} \leq X$ as explained in Fig. 5. In the following, these modules are explained 


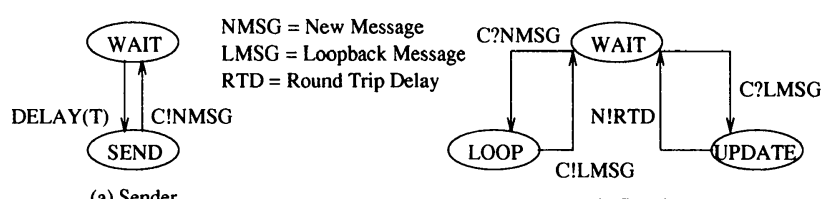

(a) Sender

(b) Receiver

Figure 6: State Diagram of the ADM Module

in detail.

\subsubsection{Actual Delay Measurement (ADM) Module}

Operation of the ADM module is explained using the state diagram in Fig. 6. The ADM protocol consists of two modules, the sender and the receiver. The sender in one $\mathrm{ADM}$ entity communicates with the receiver in another entity by interacting with the underlying service provider through the interaction point $C$. The sender sends a new message NMSG(id,time_stamp) containing every $T$ seconds, where $i d$ is an identifier for the $\mathrm{ADM}$ at one end and time_stamp contains the time of sending the message. The receiver module receives two kinds of messages $N M S G$ and $L M S G$. An $N M S G$ message is generated by the sender at the other ADM module as explained above. When the receiver receives an $N M S G$, the message is simply sent back to the sender as a loopback message $L M S G$. When a loopback message $L M S G$ is received, the receiver computes the Round Trip Delay (RTD) for that message, by subtracting the time_stamp in the message from the current time, and sends the RTD to the Future Delay Estimation module through the interaction point $N 1$.

There are two important characteristics of the delay measurement protocol which make the protocol very simple. First, since only the sender puts a time stamp on an outgoing message and the receiver simply returns the message, only the local clock at the sender is sufficient to compute the round trip delay. Since no global clock is involved, clock synchronization problems do not arise and delay measurement becomes a local computation. Second, there is no acknowledgement policy in the protocol because the protocol's objective is not to provide a reliable data transfer service. The loss of a message in either direction means that a delay sample for the FDE module is lost implying that the delay samples are not generated periodically. Any kind of disturbance in the periodicity of the delay samples, which are used to train the neural network in the FDE module, is not of any consequence because neural networks have the ability to obtain generalized behavior from data patterns containing disturbances [WASS 89].

\subsubsection{Request Processing (RP) Module}

The request processing module is an interface module that receives a request, $G E T \_R T D$, for an estimation of the round trip delay in the service provider. This module sends the request to the FDE module through the $N 2$ interaction point and when the FDE module sends an estimation of the delay back, a reply is sent to the user. The purpose of the RP module is to free the users from the details of making requests to the FDE module. 


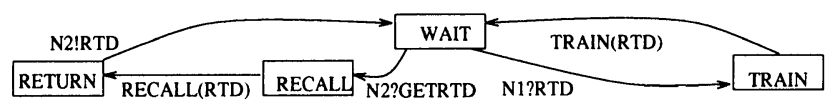

Figure 7: State Diagram of the FDE Module

\subsubsection{Future Delay Estimation (FDE) Module}

Operation of the FDE module is explained using the state diagram in Fig. 7. This module receives messages from the $\mathrm{ADM}$ and $\mathrm{RP}$ modules through the interaction points $N 1$ and $N 2$, respectively. Initially, the module is in the IDLE state. A Round Trip Delay (RTD) message received at the $N 1$ interaction point, denoted by the transition N1?RTD, takes the module to the state TRAIN. In state TRAIN, the FDE module trains the neural network using the new round trip delay sample RTD received at interaction point N1. This training of the neural network is shown by the internal action TRAIN(RTD) in the TRAIN state.

In the WAIT state, the arc with the label N2?GETRTD means that the FDE module can receive a message GETRTD (Get Round Trip Delay) from the RP module through the interaction point N2. The GETRTD message is a request to the FDE module from a user to get the latest estimation of the round trip delay stored in the neural network. Upon receiving a GETRTD message, the FDE module moves to the RECALL state. In the RECALL state, an internal action RECALL(RTD) is performed, that does a recall operation on the neural network to extract the current estimate of round trip delay. The RECALL(RTD) takes the FDE module to the RETURN state in which the message RTD is output to the RP module through the interaction point $\mathrm{N} 2$.

\subsection{Example of Delay Estimation Using Neural Network}

We demonstrate the usefulness of neural networks in estimating delays by simulating the behavior of the FDE module using the Neural Network Toolbox (NNTbox) subsystem of the MATLAB [MAT 92] package. MATLAB allows a user to define the kind of neural network one wants to use, the number of layers and the number of neurons in each layer, the learning rate, the sum-squared error goal, and the number of iterations (epochs) to be used in training the network.

The MATLAB program simulating the behavior of the FDE module consists of three parts: parameter initialization, initial training of the network with a given set of measured delays, and retraining of the network with each additional measured delay. As a part of initialization, we considered a 2-layer backpropagation [RUHI 86] network with 10 neurons in the first layer. Since our requirement had one input line and one output line, the second layer consisted of a single neuron. The sum-squared error goal was set at 0.02 , the learning rate at 0.02 , and the maximum epoch at 10000 . (These parameters were set after a few experiments with a given set of input/output data representing measured delays.) A set of 20 arbitrary delay values between 100 and 500 time units were chosen to initially train the network. Then, the network was retrained for each additional measured delay value for four instances.

A graph with an actual delay curve and the predicted delay curve is shown in Fig. 8. 


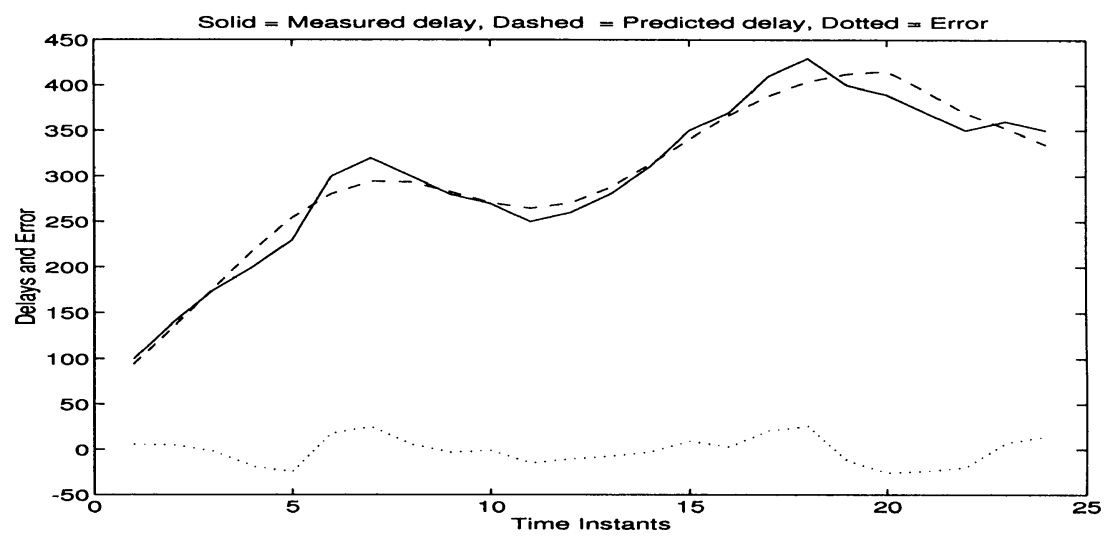

Figure 8: Comparison of predicted delay with measured delay.

The graph shows that it is possible to predict a very close approximation of the actual delay, with error less than $10 \%$, from a set of measured delays.

The simulation was run on a single processor Sun-4 workstation. The program took about 225 seconds to learn the function representing the initial 20 delay values and between 2 to 10 seconds to incorporate each additional delay value into the delay function. Thus, it is realistic to measure the delay in a service provider network and train the neural network every few seconds.

\section{Properties of a Reliable Test Architecture}

Definition: A test architecture is defined to be reliable if the test verdict, that is the result of testing the $I U T$ using $t$, depends on $t$ and IUT only.

Conceptually, the idea behind a reliable architecture is to be able to assess the correctness of a protocol implementation, with respect to a test case, independent of the environment, that is, the behavior of the service provider, the speed of testing, and the execution of the previous test cases. In reality, while testing an implementation using an external test architecture, the following behaviors can influence the result of testing.

(i) Because of errors in the IUT, connections between the LT and IUT may be prematurely disconnected during the testing process leaving many unknowledged messages in the service provider.

(ii) While testing high-speed network protocols, the service provider may contain a large number of such unacknowledged messages.

(iii) In an automated test execution environment, where hundreds of test cases are executed one after another with a very small time gap between the (un)successful termination of one test case and the initiation of the next test case, presence of unacknowledged messages in the service provider from one test execution may influence the execution of subsequent tests. 
In the following, we introduce some notations and definitions to state the properties of a reliable test architecture and discuss mechanisms to obtain test architecture reliability.

We denote a test suite $T$ as a collection of test cases $\left\{t_{1}, t_{2}, \ldots, t_{n}\right\}$. The execution of test case $t_{j}$ after the execution of test case $t_{i}$ is denoted by $t_{j}>t_{i}$, and the execution of $t_{j}$ before the execution of a test case $t_{i}$ is denoted by $t_{j}<t_{i}$.

The set of events sent by the Lower Tester to the IUT during the execution of a test case $t_{i}$ is denoted by $\operatorname{LT}\left(t_{i}\right)$ and the set of events sent by the IUT to the LT during the execution of $t_{i}$ is denoted by $I U T\left(t_{i}\right)$. It may be noted that we don't use a similar term for the UT, because the UT directly interacts with the IUT and not through the service provider.

Definition: An event $e$ is said to be a spurious event with respect to the execution of a test case $t_{i}$ if $e \in\left\{L T\left(t_{j}\right) \cup I U T\left(t_{j}\right)\right\}$ for any $t_{j}<t_{i}$.

Intuitively, while testing an erroneous IUT in an external architecture using long-haul networks, premature termination of connections between LT and IUT will give rise to many spurious events in the service provider if another test case is executed immediately after the premature termination of the previous test case. If the LT or the IUT receives spurious events during the execution of a test case $t_{i}$, then the result of testing the IUT using $t_{i}$ will be different from the actual test result without spurious events. While testing the IUT using $t_{i}$, in order for the result of testing (i.e. the test verdict) to be independent of the spurious events, it is essential for the test architecture to satisfy the following two conditions.

P1: The IUT must not take any action in response to events $e \in L T\left(t_{j}\right) \forall\left(t_{j}<t_{i}\right)$.

P2: The LT must not take any action in response to events $e \in I U T\left(t_{j}\right) \forall\left(t_{j}<t_{i}\right)$. Conditions P1 and P2 can be met in two ways:

(i) By closing connections in a graceful manner during the testing process.

(ii) By detecting and ignoring spurious events.

In order to gracefully close a connection, LT and IUT must know that the other side has received all the events sent and must wait to receive acknowledgement for those events. This implies that the following two conditions must be satisfied:

C1: An entity (LT or IUT) must not close a connection until it has received all of the other entity's (IUT's or LT's) transmitted events and can respond to them.

C2: An entity (LT or IUT) must not close a connection until it has received an acknowledgement for all its transmitted events or allowed time for an acknowledgement of its final event to return.

Though a protocol specification can be designed to gracefully close a connection during normal operation, it may not be possible to satisfy conditions $\mathrm{C} 1$ and $\mathrm{C} 2$ during the testing phase because of two reasons. First, an IUT might contain several errors to prevent a connection to be gracefully closed and second, in case of a failure of the service provider, it is not possible to gracefully close a connection. Therefore, we must have additional mechanisms in test architectures to detect and ignore spurious events. In the following, we discuss two ways to detect spurious events.

(a) Event Identifier (EID) Approach: In this approach, each time a test case is executed, unique identifiers are associated with the transmitted events generated by both the Lower Tester and the IUT such that events belonging to old connections have improper sequence numbers. By improper sequence we mean sequence numbers less than 
the sequence number of the first event sent out in the current test execution. Unique identifiers for events can be generated by choosing a unique identifier for the first event and then incrementing the identifier for each subsequent event similar to the technique used in [BELS 76]. A real-time clock in the host can be used to select the initial sequence number that is treated as a message identifier. The disadvantages with this scheme are the followings.

(i) Not all events generated by all protocols can be identified by sequence numbers. For example, the CR, CC, DR, and DC events in the ISO transport protocol do not have sequence numbers. Thus, adding a sequence number field to those events requires redesigning the protocol, which may not be desirable.

(ii) Since the IUT may contain several errors, there is no guarantee that it generates unique identifiers for the events.

(iii) Generating initial unique identifiers using real-time clocks at two entities involves real-time clock synchronization.

Therefore, it is not a good idea to build any spurious event detection mechanism into the LT and the IUT. Any such mechanism must be external to both L'T and IUT as discussed below.

(b) Block Identifier (BID) Approach: The basic idea in this approach is to treat all the events exchanged, between L'T and IUT, during the execution of a test case as a block of events and associate a unique identifier with the block. The salient features of this approach are the followings:

(i) Conditions P1 and P2 are satisfied.

(ii) The spurious event detection mechanism is external to the test entities LT and U'T, the $\mathrm{IU}^{\prime} \mathrm{T}$, and the service provider.

(iii) The test process is unaffected even by repeated execution or abortion of the same test case.

A block identifier-based mechanism to detect spurious events is shown in Fig. 9. A traditional test architecture has been augmented with two external filter protocol entities. The filter protocol entities, in cooperation with the test entities LT and UT, detect and discard spurious events. Functions of the test entities in the detection of spurious events are minimal and are restricted to informing the filter protocols of the begin and end of test cases. Before executing a test case, one of the test entities LT and UT issues a Begin of Test (BOT) and when the execution of the test case is completed or aborted, one of the test entities issues an End of Test (EOT) event to the associated filter protocol entity. Apart from informing the filter protocols of the begin and end of the execution of a test case, the LT and UT have no other role in the mechanism to detect spurious events. Every time a test case is executed, either as a new test case or to executc some test case repeatedly, a pair of BOT and EOT are generated by the test entities. All the events exchanged between the LT and the IUT after a BOT and before an EOT are treated as an indivisible block of events by the filter protocol entities, which assign a unique block identifier to all the events in the block. In the beginning, the filter protocols use an initially agreed upon unique identifier, but after the first block of events the two filter protocol entities negotiate the next unique block identifier.

Operation of the filter protocol is explained in Fig. 10. The protocol has six states and 13 transitions. After setting up the initial Block Number (BN), the protocol moves 


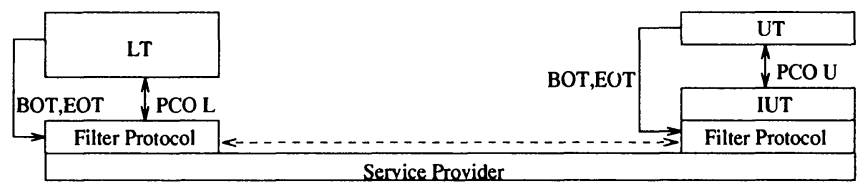

Figure 9: Using an Event Filter Protocol to Discard Spurious Events

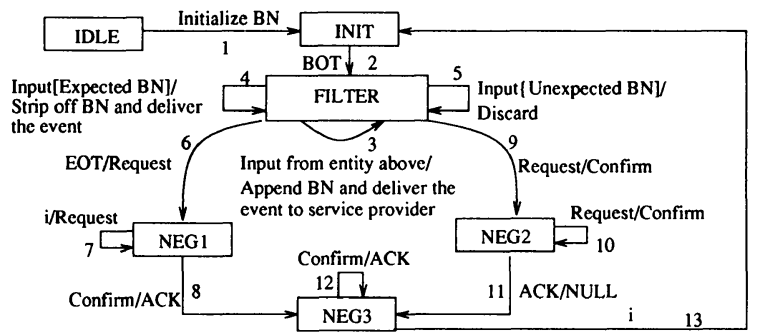

Figure 10: Operation of the Filter Protocol

from IDLE state to INIT state. A BOT event from one of the test entities LT and UT takes the protocol to the FILTER state.

The filter protocol stays in the FILTER state throughout the execution of a test case. Three kinds of messages can be received in the FILTER state: an EOT message from LT/UT, service primitives from LT and IUT, and service primitives from the service provider. Transition 3 explains that the protocol appends the current block number to the service primitive received from LT or IUT and delivers the new primitive to the service provider. According to transition 4, if a message with the expected block number is received from the service provider, the protocol strips off the block number and delivers the service primitive to the entity above. However, if the block number in the message received from the service provider is not the expected one, then the message is discarded, as shown in transition 5 .

When the execution of a test case is normally completed or aborted, one of the test entities LT/UT sends an EOT message to the associated filter protocol entity. That is, at the end of a test case execution, the filtcr protocol comes out of the event filtering function in state FILTER and enters into a negotiation mode to determine the next unique block number. Negotiation of the next unique block number is done using the three states NEG1, NEG2, and NEG3, and the transitions 6 through 13. Negotiation of the next unique block identifier is done in a manner similar to the three-way handshake mechanism of transport protocol [BOCH 89] and is explained below.

After receiving an EOT message from LT/UT in the FILTER state, the protocol sends a Request for block number negotiation to the peer filter entity through the service provider, as explained in transition 6. If a Confirm is not received from the peer entity 


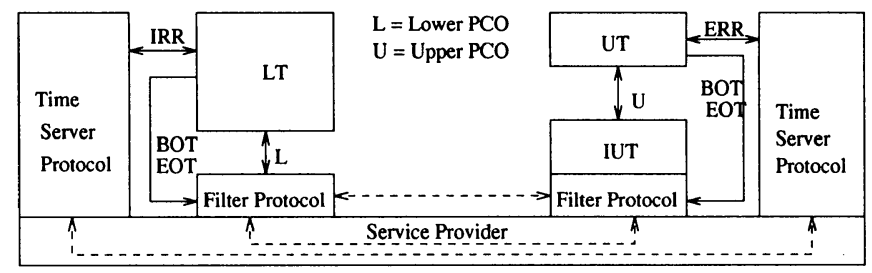

Figure 11: A Reliable DS Architecture for 'Testing Timed Behavior

during an expected time interval, the Request is sent out a few times more as shown by transition 7, where $i$ represents an abstraction for an internal decision made while sending the Request once more. If a Confirm is received in state NEG1, the protocol entity sends an acknowledgement (ACK), shown in transition 8 , to its peer and moves to NEG3 state.

Since an EOT can be generated by either the LT or the UT, the filter protocol must be ready to accept a Request for block number negotiation in the FILTER state. Receiving a Request in the FILTER state indicates that execution of the test case will no more proceed. Therefore, a filter entity sends a Confirm message to the peer entity, as shown in transition 9, and moves to state NEG2. If a Request is received once more, a Confirm is once again sent out as shown in transition 10. However, if an ACK is received, the protocol entity moves to NEG3 state as shown in transition 11. State NEG3 denotes that a unique block number has been negotiated between the two filter protocol entities. The protocol moves from the NEG3 state to the INIT state, denoted by transition 13, after some internal actions representing the updation of local variables in the filter protocol.

The function denoted by states NEG1, NEG2, and NEG3 and transitions 6 through 13 represent only the desired behavior in the block number negotiation phase. Invalid behavior generated in this phase are not shown in the state diagram for simplicity. Generation of the EOT and BOT will be discussed in Section 5 .

\section{Reliable Test Architecture for Timed Behavior}

By combining the ideas presented in Section 3 and 4 with the basic test architecture in Fig 1, we obtain the reliable architecture in Fig. 11. A reliable test architecture has the following features.

(i) The points of control and observation of the basic test architectures remain unchanged in the new architecture. Therefore, the principles of testing using different architectures such as DS, CS, and RS are preserved.

(ii) The LT and UT, while initiating a timer, make a request to the time-server protocol to obtain the current best estimate of the round-trip delay in the service provider.

(iii) The filter protocol entities negotiate a new block number in the interval between the execution of two consecutive test cases. Since these entities do not generate any new message while a test case is running, operation of the filter protocol is transparent to the execution of test cases.

In order to use good estimates of timer intervals in the test entities and achieve 


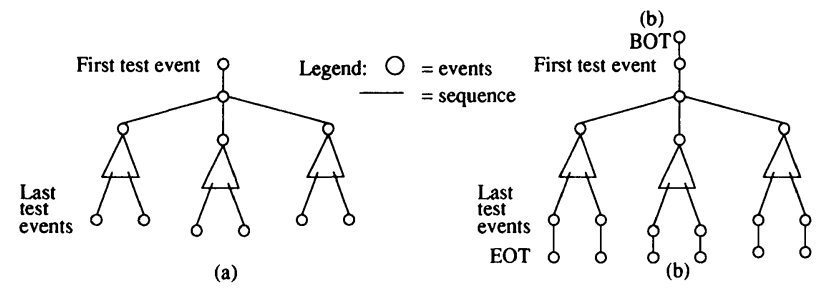

Figure 12: Introduction of BOT and EOT events in Test Cases

reliability in testing, the dynamic behavior of test cases must interact with the timerserver protocol and filter protocol entities. For this purpose, the dynamic behavior part of test cases need to be augmented in the following two ways.

Interacting with the time-server protocol: The syntax of initiating a timer in TTCN is: STARTTIMER(id,duration), where id is an identifier for the timer and duration is the interval of the timer after which the timer, if still active, generates a timeout event. The duration of a timer depends on the purpose of the timer as explained in Section 2. But, an important factor in deciding the value of a timer is the present round-trip delay in the service provider. 'Therefore, before initiating a timer, a test case must obtain the current estimate of the round-trip delay in the service provider. Therefore, while initiating a timer, a test designer can use the following syntax:

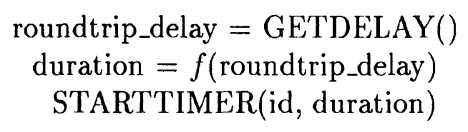

The call GETDELAY() interacts with the Request Processing module of the time-server protocol, explained in Section 3.2.2, and obtains the current estimate of the round trip delay in the service provider. The duration of a timer is computed from the roundtrip_delay using some function $f$ that depends on the purpose of the timer and is decided by the test designer. In case of a retransmission timer, the duration is typically computed as duration $=$ roundtrip_delay $+\Delta$, where $\Delta$ is the time assumed to be taken by the IUT to generate an acknowledgement.

Interacting with the Filter Protocol: The Lower Tester interacts with the filter protocol for two reasons: first, to inform the filter protocol of the beginning and end of a test case execution by sending EOT and BOT events and second, to exchange regular test events through the lower PCO (L). The Upper Tester interacts with the filter protocol only to communicate the BOT EOT events to the filter protocol, because in some test cases the Upper Tester initiates the execution of tests. The introduction of BOT and EOT events in a test case is illustrated in Fig. 12. Conceptually, a TTCN test case can be viewed as a tree of test events [ISO 9646] with a start test event and a set of possible end test events, as shown in Fig. 12(a). Depending on the response of the IUT, the 
test case executes one sequence of test events leading from the first to one of the many end test events. Therefore, to communicate the beginning of a test case to the filter protocol, we add a new event BOT before the first event and to communicate the end of test execution, an EOT is added after each end test event, as shown in Fig. 12(b).

\section{Conclusions and Further Work}

We presented the notion of a time-server that dynamically estimates the service provider's round-trip delay using neural network technique. We introduced the notion of test architecture reliability, defined the properties of a reliable architecture, and presented the design of a filter protocol to detect and discard spurious test events to achieve architecture reliability. The work presented in this paper provides a better means of testing by correctly estimating timer intervals in test cases and by isolating the execution of one test case from the execution of previous test cases. Additional research needs to be done to estimate the frequency of actual delay measurement so that the real delay in the service provider is not affected.

\section{References}

[ALKA 90] N. P. Alfano and B. Kanungo, "Development of an International Standard for Conformance Testing X.25 DTEs," Proc. of the 10th. International Symposium on Protocol Specification, Testing, and Verification, Ottawa, June 1990, pp. 121-132.

[BELS 76] D. Belsnes, "Single-Message Communication," IEEE Trans. on Comm., VOL. COM-24, NO. 2, February 1976, pp. 190-194.

[DSU 90] A. T. Dahbura, K. K. Sabnani, and M. U. Uyar, "Formal Methods for Generating Protocol Conformance Test Sequences," Proceedings of the IEEE, Vol. 78, No. 8, August 1990, pp. 1317-1326.

[GROS 88] S. Grossberg (Editor), Neural Networks and Natural Intelligence, The MIT Press, 1988.

[HECH 88] R. Hecht-Nielsen, "Applications of Counter Propagation Networks," Neural Networks 1:131-139.

[ISO 9646] ISO/IEC 9646: Information Technology - Open Systems Interconnection Conformance Testing Methodology and Framework, 1991.

[KOHO 88] T. Kohonen, Self-organization and associative memory, 2d ed. New York: Springer Verlag, 1988.

[MAT 92] MATLAB Reference Guide 1992, The MathWorks Inc., USA.

[NASA 92] K. Naik and B. Sarikaya, "Protocol Test Design and Verification," IEEE Software, January 1992, pp. 27-37.

[RUHI 86] D. E. Rumelhart, G. E. Hinton, and R. J. Williams, "Learning internal representations by error propagation," in Parallel distributed processing, Vol. 1, pp. 318-362, MA: MIT Press.

[WASS 89] P. D. Wasserman, Neural Computing: Theory and Practice, Van Nostrand Reinhold, New York, 1989.

[ZHAN 86] L. Zhang, "Why TCP Timers Don't Work Well?," Proc. of the Tenth Data Communications Symposium, Montreal 1986. 